\title{
CARDIOTOCOGRAFIA EM GeSTAÇÕES COM DIÁSTOLE ZERO OU REVERSA NAS ARTÉRIAS UMBILICAIS: ANÁLISE DOS RESULTADOS PERINATAIS
}

\author{
Rosel Mieko Yamamoto Nomura*, Rossana Pulcineli Vieira Francisco, Seizo Miyadahira, Marcelo Zugaib. \\ Trabalho realizado no setor de Vitalidade Fetal-Clínica Obstétrica-Hospital das Clínicas \\ da Faculdade de Medicina da Universidade de São Paulo, SP
}

RESUMO - OBJEtivos. Estudar, em gestações com diástole zero (DZ) ou reversa (DR), dopplervelocimetria das artérias umbilicais os resultados perinatais de acordo com os parâmetros cardiotocográficos.

Métodos. Entre 1993 e 2000, foram selecionadas 127 gestantes com DZ ou DR. A classificação adotada para a cardiotocografia foi ativo (normal), hipoativo (suspeito) e inativo (alterado) Os parâmetros analisados foram: variabilidade da FCF, aceleraçóes transitórias, DIP II, DIP umbilical, desaceleração prolongada e padrão pseudo sinusoidal.

Resultados. Houve associação $(p<0,05)$ entre o padrão inativo e acidemia no nascimento (pH inferior a 7,20) em 71,9\%, Apgar de $I^{\circ}$ minuto inferior a sete $(73,4 \%)$, intubação do $\mathrm{RN}(64,1 \%)$, e óbito neonatal precoce $(20,3 \%)$. Houve associação $(p<0,05)$ entre a ausência de acelerações transitórias com acidemia no nascimento (58,8\%), Apgar de $1^{\circ}$ minuto inferior a sete $(67,7 \%)$, intubação do RN (51\%) e total de óbitos no berçário $(29,4 \%)$. A redução da variabilidade $(<5 \mathrm{bpm})$ associou-se a $(p<0,05)$ : acidemia no nascimento $(88,5 \%)$, intubação do RN $(69,2 \%)$, óbito neonatal precoce $(34,6 \%)$ e total de óbitos no berçário (42,3\%). Constatou-se associação entre a ocorrência de DIP II $(p<0,05)$ e a acidemia no nascimento em 78,2\%. 0 DIP umbilical grave associou-se $(p<0,05)$ com acidemia no nascimento $(79,3 \%)$, intubação do recém-nascido $(69 \%)$ e óbito neonatal precoce $(17,2 \%)$. Houve associação $(p<0,05)$ entre a desaceleração prolongada e intubação do RN $(70,6 \%)$. 0 padrão pseudo sinusoidal associou-se $(p<0,05)$ com 0 óbito neonatal precoce $(60 \%)$.

ConclusöEs. A DZ ou DR representa grave comprometimento fetal, cujo risco para óbito neonatal ou morbidade perinatal é extremamente elevado e constata-se correlação entre as alterações da cardiotocografia com os resultados perinatais adversos.

UnITERMOS: Diástole zero. Diástole reversa. Cardiotocografia. Resultados perinatais.

\section{INTRODUÇÃO}

A dopplervelocimetria tem sido amplamente utilizada em obstetrícia para se avaliar a função placentária, por meio de estudos da velocidade de fluxo sangüíneo nos vasos do cordão umbilical ${ }^{1,2}$. Nas gestações que evoluem com deterioração da função placentária, 0 aumento da resistência ao fluxo sangüíneo é evidenciado pela redução da velocidade diastólica nas artérias umbilicais ${ }^{3}$. Nos casos graves observa-se ausência de fluxo diastólico final (Diástole Zero) ou o fluxo reverso na diástole (Diástole Reversa), o que representa comprometimento extremo da função placentária ${ }^{4,5}$, responsável por elevadas taxas de mortalidade e morbidade perinatais, ${ }^{6,7}$.

$\mathrm{Na}$ Clínica Obstétrica do Hospital das Clí-

\begin{tabular}{c} 
*Correspond ência: \\
\hline Rua General Canavarro, 280 Bairro Campestre \\
Santo André-SP-CEP 09070-440 \\
Tel:(011)4991 2481 / 4221 8778 \\
FAX:(011)4991-7312/42218752 \\
E-mail:roseli.nomura@terra.com.br
\end{tabular}

nicas da Faculdade de Medicina da Universidade de São Paulo (HC-FMUSP), no período compreendido entre 1992 e 1999, observouse, em casos de Diástole zero (DZ) ou Diástole reversa (DR), mortalidade perinatal de $35,8 \%{ }^{8}$. Em razão desses fatos, as gestantes com este diagnóstico devem ser referidas à centros hospitalares com estrutura de cuidados terciários. A aplicação de metodologia de avaliação do bem-estar fetal dotada de grande acurácia é imperativa nesses casos, pois ela é essencial para se evitar ou minimizar os freqüentes danos fetais oriundos da carência do aporte de oxigênio. Para tanto, o diagnóstico precoce desta condição é imprescindível para propiciar intervenções oportunas, porque em sua maioria ocorrem situações de prematuridade extrema, a qual continua a desafiar a neonatologia, apesar dos grandes avanços aí observados 9.10 .

A cardiotocografia, um dos exames mais utilizados na avaliação do bem-estar fetal, é o mais importante no seguimento dessas gestações ". Baseia-se no comportamento da fre- qüência cardíaca fetal (FCF) e é efetuado por meio de dispositivo especial (cardiotocógrafo), o qual registra em traçados próprios a FCF, as contrações uterinas e os movimentos corpóreos fetais.

Os padrões alterados da cardiotocografia, sob influência do Sistema Nervoso Autônomo, expressam os episódios de hipoxemia fetal, os quais também desencadeiam fenômenos adaptativos caracterizados pela vasodilatação dos territórios considerados nobres (cérebro, coração e adrenais) e vasoconstrição na circulação de outros órgãos e sistemas com detrimento da circulação periférica fetal ${ }^{12}$. Ao mesmo tempo, através dos reflexos cardiovasculares mediados pela estimulação dos quimiorreceptores, o sistema nervoso autônomo promove alterações nas características da FCF que são evidenciadas pela cardiotocografia $^{13}$. Conforme o déficit da oxigenação se agrava, diferentes padrões cardiotocográficos podem ser observados.

Ao contrário do que ocorre nas gestações próximas ao termo, os casos de DZ ou 
$D R$, em função da tenra idade gestacional em que freqüentemente são acometidos, impõem dificuldades na interpretação dos diversos parâmetros cardiotocográficos ${ }^{14}$. Considerando-se a gravidade da insuficiência placentária envolvida, cada detalhe do exame torna-se muito importante na condução destas gestações, tendo como escopo a eleição do melhor momento para a intervenção obstétrica resolutiva.

Ao aceitar como verdadeiro o pressuposto de que a ocorrência de DZ ou DR nos sonogramas das artérias umbilicais revela a insuficiência placentária grave, a Clínica Obstétrica da FMUSP dedica especial atenção a esses casos, reservando-lhes cuidados muito especiais. A propedêutica fetal é aplicada de forma intensiva, especialmente a cardiotocografia, que é realizada diariamente, ou em menores intervalos de tempo, conforme prevêo protocolo assistencial assim estabelecido neste serviço há quase uma década. Em torno dessa propedêutica avaliando o bem-estar fetal, orbitam os pilares que modulam a conduta obstétrica: a idade gestacional, o peso fetal estimado e a maturidade fetal.

Tendo acumulado importante casuística neste incomum contingente obstétrico, 0 presente trabalho propõe analisar minuciosamente a cardiotocografia abrangendo, em sua plenitude, os vários parâmetros que a compõem, tendo como contraponto os resultados perinatais.

\section{Métodos}

No período compreendido entre maio de 1993 eabril de 2000, o setor de Vitalidade Fetal da Clínica Obstétrica do Hospital das Clínicas da Faculdade de Medicina da Universidade de São Paulo (HC-FMUSP) acompanhou 249 gestações de alto risco, nas quais constatou-se DZ ou DRà dopplervelocimetria das artérias umbilicais. Todas pacientes foram hospitalizadas para seguimento rigoroso da vitalidade fetal, tratamento clínico e obstétrico.

Foram selecionados 127 casos com traçados cardiotocográficos disponíveis para revisão. Nesta análise, considerou-se apenas o último traçado realizado nas 24 horas que antecederam o momento do parto, realizado no HC-FMUSP. Foram excluídos 54 casos $(21,7 \%)$ com dados incompletos de gasometria da artéria umbilical no nascimento, 33 de óbito fetal (13,3\%), I 8 com traçado cardiotocográfico indisponível para revisão $(7,2 \%)$, nove com anomalias fetais $(3,6 \%)$ e oito gestações gemelares $(3,2 \%)$. Em relação aos antecedentes obstétricos, 43 pacientes $(33,9 \%)$ eramnulíparas, e84 (66, I\%) eram multíparas. A média da idade materna apresentou valor de 29,4 anos, com desvio padrão de seis anos. Quanto às intercorrências clínicas e obstétricas associadas, constatou-se, com maior freqüência, a ocorrência de síndromes hipertensivas (I0I casos, $79,5 \%$ ), seguida pela cardiopatia materna ( 13 casos, 10,2\%) e diabete melito (nove casos, $7,1 \%$ ).

Adopplervelocimetria das artérias umbilicais foi realizada por via transabdominal, utilizando-se dos seguintes equipamentos de ultra-sonografia: ATLUltramark9 ou ATL HDI 3000 (Advanced Technology Laboratories Inc., Bothell, USA), e Toshiba SSA-340A (Toshiba Co., Otawara, Japan). Os exames foram realizados com a paciente em posição semi-sentada. Os sonogramas foram obtidos em períodos de inatividade fetal (ausência de movimentos corpóreos e respiratórios), utilizando-se filtros de baixos valores, entre $25 \mathrm{~Hz}$ a $50 \mathrm{~Hz}$. Analisou-se o segmento do cordão umbilical próximo à placenta, aproximadamente $\mathrm{a} 5 \mathrm{~cm}$ de sua inserção. As duas artérias do cordão umbilical foram avaliadas e o diagnóstico de DZ foi confirmado quando não se observou fluxo diastólico, isto é, quando a velocidade durante a diástole tornou-se nula. A DR foi caracterizada quando observou-se fluxo negativo durante a diástole no canal inverso do sonograma, caracterizando o fluxo reverso. A insonação da artéria umbilical foi repetida em pelo menos cinco momentos diferentes durante o exame dopplervelocimétrico, ativando-se o dispositivo de Doppler, com mapeamento colorido de fluxo, por pelo menos um minuto.

Todos os traçados cardiotocográficos foram analisados visualmente para a elaboração deste trabalho. A cardiotocografia anteparto foi realizada com aparelhos da marca HewlettPackard, modelo HP 804IA(Hewlett-Packard, Boblingen, Germany). $\mathrm{O}$ exame foi realizado segundo técnica padronizada nesteSetor, com a paciente em posição de semi-Fowler, velocidade de registro gráfico de um centímetro por minuto e o traçado realizado por, no mínimo, 30 minutos.
Os seguintes parâmetros cardiotocográficos foram analisados: classificação da cardiotocografia pelo índice cardiotocométrico, presença de acelerações transitórias, variabilidade da FCF, desacelerações tardias (DIP II), desacelerações variáveis (DIP umbilical), desacelerações prolongadas e padrão pseudo sinusoidal. A classificação baseou-se nos critérios e definições estabelecidos pelo National Institute of Child Health and Human Development Research Planning Workshop, 199715. A variabilidade da FCF foi analisada de acordo com a observação das oscilações da linha de base e foi categorizada em inferior a $5 \mathrm{bpm}$, entre 5 e $9 \mathrm{bpm}$ e superior ou igual a $10 \mathrm{bpm}$. As acelerações transitórias foram caracterizadas como ascensos da FCF, com amplitude mínima de 15 bpm e com duração superior a 15 segundos, independente da idade gestacional. Os traçados foram classificados de acordo com a presença ou não de, no mínimo, uma aceleração transitória. Foi caracterizada como desaceleração tardia (DIP II) a queda gradual da FCF, com intervalo entre início da queda e nadir superior a 30 segundos, relacionada à contração uterina e com decalagem longa, superior a 20 segundos. As desacelerações variáveis (DIP umbilical) foram categorizadas em: leve (queda não atingindo FCF de 80 bpm e com duração inferior a 30 segundos), moderada (queda atingindo FCF entre 70 e 80 bpm e/ou duração entre 30 e 60 segundos) ou grave (queda abaixo de $70 \mathrm{bpm}$ ou superior a 60 segundos). Na ocorrência de mais de um tipo de desaceleração variável foi considerada somente a de maior gravidade. $A$ desaceleração prolongada foi caracterizada quando a queda da FCF, relacionada ou não à contração uterina, apresentava-se rápida ou lenta, de amplitude variável, porém com duração superior a dois minutos. O padrão pseudo sinusoidal foi caracterizado quando o traçado apresentava oscilações, com ondas uniformes de amplitude variando de 5 a $15 \mathrm{bpm}$, entretanto, não como o padrão sinusoidal clássico, mas com morfologia do traçado semelhante.

A cardiotocografia foi classificada também de acordo com oíndice cardiotocométrico de Zugaib e Behle ${ }^{16}$, sendo o feto classificado em ativo (vitalidade normal), hipoativo (vitalidade suspeita) ou inativo (vitalidade alterada).

Foram analisados os seguintes resultados perinatais: idade gestacional no nascimento, $\mathrm{pH}$ 
Tabela I - Médias e desvios padrão da idade gestacional no nascimento de acordo com os parâmetros cardiotocográficos, em gestações com diástole zero ou reversa

\begin{tabular}{|c|c|c|c|}
\hline \multirow[t]{2}{*}{ Parâmetro } & \multicolumn{2}{|c|}{ Idade gestacional no nascimento } & \multirow[b]{2}{*}{$\mathrm{p}$} \\
\hline & Média & DP & \\
\hline \multicolumn{4}{|c|}{ Classificação da cardiotocografia } \\
\hline Ativo & 32,87 & 1,88 & $\mathrm{~F}=5,83 * *$ \\
\hline Hipoativo & 31,44 & 2,23 & $p<0,003$ \\
\hline Inativo & 31,02 & 2,48 & \\
\hline \multicolumn{4}{|l|}{ AT } \\
\hline presente & 32,87 & 1,88 & $0,001 *$ \\
\hline ausente & 31,18 & 2,39 & \\
\hline \multicolumn{4}{|l|}{ Variabilidade } \\
\hline$<10 \mathrm{bpm}$ & 31,00 & 2,47 & $0,01 *$ \\
\hline$\geq 10 \mathrm{bpm}$ & 32,07 & 2,17 & \\
\hline \multicolumn{4}{|l|}{ Desaceleração } \\
\hline presente & 31,34 & 2,36 & $0,09 *$ \\
\hline ausente & 32,25 & 2,39 & \\
\hline \multicolumn{4}{|l|}{ DIP II } \\
\hline presente & 31,47 & 2,34 & $0,87^{*}$ \\
\hline ausente & 31,54 & 2,43 & \\
\hline \multicolumn{4}{|l|}{ DIP umbilical } \\
\hline presente & 31,24 & 2,27 & $0,08 *$ \\
\hline ausente & 32,01 & 2,55 & \\
\hline \multicolumn{4}{|l|}{ Desaceleração prolongada } \\
\hline presente & 30,25 & 2,58 & $0,02^{*}$ \\
\hline ausente & 31,70 & 2,31 & \\
\hline \multicolumn{4}{|l|}{ Padrão pseudo sinusoidal } \\
\hline presente & 31,17 & 2,40 & $0,75^{*}$ \\
\hline ausente & 31,52 & 2,39 & \\
\hline
\end{tabular}

* testet deStudent, ${ }^{* *}$ ANOVA

da artéria umbilical no nascimento, índices de Apgar de $1^{\circ} \mathrm{e} 5^{\circ}$ minuto (alterados quando com valores inferiores a 7), intubação do recémnascido em sala de parto e a mortalidade neonatal. Aidade gestacional (IG) foi calculadaa partir da data da última menstruação (DUM), quando esta era compatível com a IG estimada pela primeira ultra-sonografia realizada, no máximo, até a vigésima semana de gestação (discordância inferior a 14 dias). Nos casos em que não houve esta concordância, utilizamos a IG calculada através da primeira ultra-sonografia. $\mathrm{OpH}$ da artéria umbilical foi avaliado em sangue colhido de segmento de cordão umbilical de cerca de $15 \mathrm{~cm}$ de comprimento, obtido por duplo clampeamento, imediatamente após onascimento. Aamostrafoi coletada em seringa previamente heparinizada, vedada para impedir contato com o ar ambiente e analisada a seguir. Valores de pH inferiores a 7,20 caracterizaram a acidemia no nascimento. A mortalidade neonatal foi analisada de acordo com a idade do recém-nascido no dia do óbito. Foi classificado como óbito neonatal precoce aqueles que ocorreram até o sétimo dia. A mortalidade durante a internação do recém-nascido no berçário foi considerada como óbito hospitalar.

Inicialmente todas as variáveis foram analisadas descritivamente. Para as variáveis quantitativas esta análise foi feita através do cálculo de médias e desvios padrão. Para as variáveis qualitativas calculou-sefreqüências absolutas e relativas. A comparação das médias dos grupos foi realizada utilizando-se o testet de Student para amostras não-pareadas, ou a análise de variância clássica (ANOVA). A comparação entreas proporçõesfoiavaliadaatravés do teste qui-quadrado ou teste exato de Fisher. O nível de significância utilizado para os testes foi de $5 \%$.

Este estudo foi aprovado pela Comissão de Ética para Análise de Projetos de Pesquisa (CAPEPesq) da diretoria clínica do HC-FMUSP.

\section{Resultados}

Foram analisados 127 casos. Trinta e sete gestações (29,5\%) apresentaram, na dopplervelocimetria das artérias umbilicais, 0 diagnóstico de diástole reversa. Em muitos casos houve associação de indicações para a resolução obstétrica (cesárea em 100\%), sendo que as mais freqüentes foram: sofrimento fetal anteparto $(60,7 \%)$ caracterizado por alterações na cardiotocografia ou no perfil biofísico fetal, diagnóstico de maturidade fetal (33,8\%), mecônio no líquido amniótico $(19,7 \%)$, piora do quadro clínico materno (I0,3\%) e oligohidrâmnio (9,5\%).

Aidade gestacional no nascimento variou de 26 semanas e 6 dias a 36 semanas e 4 dias. $\mathrm{Na}$ análise dos parâmetros cardiotocográficos (Tabela I), podemos observar que a média da idade gestacional no nascimento apresentou-se significativamente superior no grupo com cardiotocografia padrão ativo $(p=0,003)$. 0 mesmo 
Tabela 2 - Distribuição dos casos segundo os parâmetros da cardiotocografia e resultados perinatais anormais: $\mathrm{pH}$ no nascimento inferior a 7,20, Apgar de $I^{\circ}$ minuto $<7$, de $5^{\circ}$ minuto $<7$ e intubação do $\mathrm{RN}$, em gestações com diástole zero ou reversa nas artérias umbilicais

\begin{tabular}{|c|c|c|c|c|c|c|c|c|c|c|c|c|c|}
\hline \multirow[t]{2}{*}{ Parâmetro } & \multirow[t]{3}{*}{ Total } & \multicolumn{3}{|c|}{$\mathrm{pH}$ no nascimento $<7,20$} & \multicolumn{3}{|c|}{ Apgar de $1^{\circ}$ minuto $<7$} & \multicolumn{3}{|c|}{ Apgar de $5^{\circ}$ minuto $<7$} & \multicolumn{3}{|c|}{$\begin{array}{c}\text { Intubação do RN } \\
\text { no nascimento }\end{array}$} \\
\hline & & $\mathrm{N}$ & $\%$ & $p$ & $\mathrm{~N}$ & $\%$ & $p$ & $\mathrm{~N}$ & $\%$ & p & $\mathrm{N}$ & $\%$ & $p$ \\
\hline \multicolumn{13}{|l|}{ Classificação } & \\
\hline Ativo & 25 & 6 & 24,0 & & 10 & 40,0 & & 1 & 4,0 & & 5 & 20,0 & \\
\hline Hipoativo & 38 & 14 & 36,8 & $0,001 *$ & 22 & 57,9 & $0,01 *$ & 6 & 15,8 & $0,165 *$ & II & 28,9 & $0,001 *$ \\
\hline Inativo & 64 & 46 & 71,9 & & 47 & 73,4 & & 13 & 20,3 & & $4 \mid$ & 64,1 & \\
\hline \multicolumn{14}{|c|}{ Aceleração transitória } \\
\hline presente & 25 & 6 & 24,0 & & 10 & 40,0 & & 1 & 4,0 & $0,|2| * *$ & 5 & 20,0 & $0,005 *$ \\
\hline ausente & 102 & 60 & 58,8 & $0,002 *$ & 69 & 67,7 & $0,01 *$ & 19 & 18,6 & & 52 & 51,0 & \\
\hline \multicolumn{14}{|l|}{ Variabilidade } \\
\hline$<5 \mathrm{bpm}$ & 26 & 23 & 88,5 & & 20 & 70,9 & & 7 & 26,9 & & 18 & 69,2 & \\
\hline $5 \mathrm{a} 9 \mathrm{bpm}$ & 41 & 24 & 58,5 & $0,001 *$ & 27 & 65,9 & $0,09 *$ & 6 & 14,6 & $0,198 *$ & 24 & 58,5 & $0,001 *$ \\
\hline$\geq 10 \mathrm{bpm}$ & 60 & 19 & 31,7 & & 32 & 53,3 & & 7 & 11,7 & & 15 & 25 & \\
\hline \multicolumn{14}{|l|}{ Desaceleraçáo } \\
\hline presente & 103 & 59 & 57,3 & & 70 & 67,9 & & 17 & 16,5 & & 7 & 29,2 & \\
\hline ausente & 24 & 7 & 29,2 & $0,01 *$ & 9 & 37,5 & $0,006 *$ & 3 & 12,5 & $0,764 * *$ & 50 & 48,5 & $0,08 *$ \\
\hline \multicolumn{14}{|l|}{ DIP II } \\
\hline presente & 55 & 43 & 78,2 & & 37 & 67,3 & & 10 & 18,2 & & 29 & 52,7 & \\
\hline ausente & 72 & 23 & 31,9 & $0,001 *$ & 42 & 58,3 & $0,303 *$ & 10 & 13,9 & $0,510 *$ & 28 & 38,9 & $0,120 *$ \\
\hline \multicolumn{14}{|l|}{ DIP umbilical } \\
\hline grave & 29 & 23 & 79,3 & & 22 & 75,9 & & 8 & 27,6 & & 20 & 69,0 & \\
\hline moderado & 16 & 8 & 50,0 & & 11 & 68,8 & & 2 & 12,5 & & 8 & 50,0 & \\
\hline leve & 38 & 14 & 36,8 & $0,006 *$ & 22 & 57,9 & $0,262 *$ & 5 & 13,2 & $0,299 *$ & 13 & 34,2 & $0,01 *$ \\
\hline ausente & 44 & 21 & 47,7 & & 24 & 54,6 & & 5 & $\|, 4$ & & 16 & 36,4 & \\
\hline \multicolumn{14}{|c|}{ Desaceleraçãoprolongada } \\
\hline presente & 17 & II & 64,7 & & 13 & 76,5 & & 5 & 29,4 & & 12 & 70,6 & \\
\hline ausente & 110 & 55 & 50,0 & $0,25 *$ & 66 & 60,0 & $0,192 *$ & 15 & 13,6 & $0,144 * *$ & 45 & 40,9 & $0,02 *$ \\
\hline \multicolumn{14}{|c|}{ Padrão pseudo sinusoidal } \\
\hline presente & 5 & 4 & 80,0 & $0,36 * *$ & 5 & 100,0 & $0,156 * *$ & 0 & 0 & $1,0 * *$ & 3 & 60,0 & \\
\hline ausente & 122 & 62 & 50,8 & & 74 & 60,7 & & 20 & 16,4 & & 54 & 44,3 & 0,656*; \\
\hline
\end{tabular}

*Teste do Qui quadrado, ** Teste exato de Fisher

ocorrendo com os casos que apresentam aceleraçõestransitórias $(\mathrm{p}=0,00 \mathrm{I})$, variabilidadesuperior ou igual a $10 \mathrm{bpm}(\mathrm{p}=0,01)$ e na ausência de desaceleração prolongada $(\mathrm{p}=0,02)$.

$\mathrm{Na}$ Tabela 2 podemos constatar que associação significativa entre os resultados alterados na cardiotocografia e valores de $\mathrm{pH}$ de artéria umbilical no nascimento inferiores a 7,20 . Observa-se associação significativa entre a ausência de AT e a ocorrência de $\mathrm{pH}$ de artéria umbilical no nascimento inferior ou não a 7,20 ( $p=0,002)$. Na análise da variabilidade, podemos verificar associação significativa entre a redução da variabilidade e a ocorrência de $\mathrm{pH}$ de artéria umbilical no nascimento inferior a 7,20 $(p=0,00 \mathrm{I})$. Avaliando-se a presença ou não de desaceleração, a frequiência de $\mathrm{pH}$ de artéria umbilical no nascimento inferior a 7,20 foi significativamente maior $(p=0,0 I)$ quando $o$ evento esteve presente. Discriminando-se o tipo de desaceleração, podemos observar associação significativa entre a presença de DIP II $(p=0,00 \mathrm{I})$ e de DIP umbilical grave $(p=0,006)$ com os níveis de $\mathrm{pH}$ inferiores a 7,20. A ocorrência de desaceleração prolongada e do padrão pseudo sinusoidal não apresentou correlação significativa com a acidose no nascimento.

A ocorrência de Apgar de $1^{\circ}$ minuto anormal (inferior a 7) está significativamente relacionada com o padrão inativo na cardiotocografia $(p=0,0 I)$, a ausência de acelerações transitórias $(p=0,0 \mathrm{l})$ e a presença de desacelerações $(p=0,006)$ da FCF (Tabela 2). A análise do Apgar de $5^{\circ}$ minuto não revelou associação significativa entre a ocorrência de anor- malidades neste índice e a avaliação dos parâmetros da cardiotocografia.

Aintubação orotraqueal do recém-nascido nos primeiros minutos de vida está associada significativamenteaos casos com padrão inativo $(p=0,00 \mathrm{I})$, ausência de acelerações transitórias $(p=0,005)$, variabilidade inferior ou igual a $5 \mathrm{bpm}(\mathrm{p}=0,00 \mathrm{I})$, presença de DIP umbilicais graves ou moderados $(p=0,0 \mathrm{I})$ e com desaceleração prolongadafetal $(p=0,02)$ (Tabela 2).

$\mathrm{Na}$ Tabela 3 estão demonstrados os resultados da análise da cardiotocografia de acordo com a mortalidade do recém-nascido. Podemos observar que a mortalidade neonatal precoce está associada às seguintes anormalidades: cardiotocografia inativa $(p=0,00 \mathrm{I})$, variabilidade inferior a $5 \mathrm{bpm}(p=0,007)$, presença de DIP umbilical $(p=0,04)$ e padrão pseudo 


\begin{tabular}{|c|c|c|c|c|c|c|c|}
\hline \multirow[t]{2}{*}{ Parâmetro } & \multirow[t]{2}{*}{ Total } & \multicolumn{3}{|c|}{ Óbito neonatal precoce } & \multicolumn{3}{|c|}{ Total de óbitos no berçário } \\
\hline & & $\mathrm{N}$ & $\%$ & $\mathrm{p}$ & $\mathrm{N}$ & $\%$ & $\mathrm{p}$ \\
\hline $\begin{array}{c}\text { Classificaçáa } \\
\text { Ativo }\end{array}$ & 25 & । & 4,0 & & 2 & 8,0 & \\
\hline Hipoativo & 38 & 5 & 13,2 & $0,001 *$ & 9 & 23,7 & $0,051^{*}$ \\
\hline Inativo & 64 & 13 & 20,3 & & 21 & 32,8 & \\
\hline \multicolumn{8}{|c|}{ Aceleração transitória } \\
\hline presente & 25 & I & 4,0 & & 2 & 8,0 & \\
\hline ausente & 102 & 18 & 17,6 & $0,119 * *$ & 30 & 29,4 & $0,027^{*}$ \\
\hline \multicolumn{8}{|l|}{ Variabilidade } \\
\hline$<5 \mathrm{bpm}$ & 26 & 9 & 34,6 & & II & 42,3 & \\
\hline $5 \mathrm{a} 9 \mathrm{bpm}$ & $4 \mid$ & 4 & 9,7 & $0,007 *$ & II & 26,8 & $0,040 *$ \\
\hline$\geq 10 \mathrm{bpm}$ & 60 & 6 & 10,0 & & 10 & 16,7 & \\
\hline \multicolumn{8}{|l|}{ Desaceleração } \\
\hline presente & 103 & 18 & 17,5 & & 29 & 28,2 & \\
\hline ausente & 24 & I & 4,2 & $0,122 * *$ & 3 & 12,5 & $0,112^{*}$ \\
\hline \multicolumn{8}{|l|}{ DIP II } \\
\hline presente & 55 & 9 & 16,4 & & 15 & 27,3 & \\
\hline ausente & 72 & 10 & 13,9 & $0,150 *$ & 17 & 23,6 & $0,632 *$ \\
\hline \multicolumn{8}{|l|}{ DIP umbilical } \\
\hline grave & 29 & 5 & 17,2 & & 10 & 34,5 & \\
\hline moderado & 16 & 6 & 37,5 & & 7 & 43,8 & \\
\hline leve & 38 & 5 & 13,2 & $0,039 *$ & 8 & 21,1 & $0,085 *$ \\
\hline ausente & 44 & 3 & 6,8 & & 7 & 15,9 & \\
\hline \multicolumn{8}{|c|}{ Desaceleração prolongada } \\
\hline presente & 17 & 4 & 23,5 & & 4 & 23,5 & \\
\hline ausente & 110 & 15 & 13,6 & $0,284 * *$ & 28 & 25,5 & $1,0 * *$ \\
\hline \multicolumn{8}{|c|}{ Padrão pseudosinusoidal } \\
\hline presente & 5 & 3 & 60,0 & & 3 & 60,0 & \\
\hline ausente & 122 & 16 & $|3|$, & $0,024 *$ & 29 & 23,8 & $0,101 * *$ \\
\hline
\end{tabular}

*Teste do Qui quadrado, ** Teste exato de Fisher

sinusoidal $(p=0,02)$. A análise do total de óbitos no berçário e os parâmetros cardiotocográficos mostra associação significativa com casos em que as acelerações transitórias estavam ausentes $(p=0,03)$ e com os que apresentam variabilidade inferior a $5 \mathrm{bpm}(\mathrm{p}=0,04)$.

\section{Discussão}

A insuficiência placentária grave, diagnosticada pela ocorrência de diástole zero ou diástole reversa, tem sido freqüentemente associada a resultados perinatais adversos, com elevada incidência de hipoxemia e acidemia fetais $^{17}$. A evolução do padrão cardiotocográfico do feto normalmente oxigenado para o observado na hipoxemia, principalmente nas com magnitude suficiente para produzir o dano neurológico, não é bem compreendi- $\mathrm{da}^{18,19}$, dificultando o diagnóstico da anoxia fetal e conseqüente comprometimento metabólico do recém-nascido ${ }^{20}$.

A ausência de acelerações transitórias e a variabilidade reduzida, que associam-se, neste estudo, à acidemia no nascimento, podem ser resultado do sofrimento fetal. Entretanto, gestações precoces com imaturidade do sistema nervoso autônomo, principalmente antes da $32^{a}$ semana, também apresentam esta característica cardiotocográfica prejudicando a interpretação destes parâmetros ${ }^{14}$. As desacelerações prolongadas correlacionaram-se com menor idade gestacional no nascimento, provavelmente porque em gestações precoces, com comprometimento importante da circulação placentária, é freqüente a ausência de líquido amniótico favorecendo os mecanis- mos de compressão do cordão, que evoluem com a bradicardia fetal.

A análise dos resultados neonatais demonstram correlação significativa com os padrões da cardiotocografia anteparto. Nesta casuística, por se tratar de gestações longe do termo nas quais a conduta conservadora é a regra, com exceção aos casos de franco sofrimento fetal por outros exames que não 0 diagnóstico efetuado exclusivamente pela $D Z$ ou DR, é compreensível encontrar-se maior proporção de alterações graves na cardiotocografia. Nos fetos maiores, acima de 3 I semanas, a conduta ativa para resolução da gestação é muito mais freqüente. Nestas situações, os casos em questão não chegam a apresentar alterações cardiotocográficas de maior gravidade. 
No presente estudo, a análise da relação entre os parâmetros da cardiotocografia anteparto e a ocorrência de valores de $\mathrm{pH}$ no nascimento inferiores a 7,20 demonstrou associação significativa entre a acidemia e as alterações no índice cardiotocométrico, ausência de acelerações transitórias, variabilidade da FCF, ocorrência de desacelerações, DIPsIl, e DIPs umbilicais graves. É interessante notar que, apesar de não atingir significância estatística, existe menor proporção de acidemia no grupo com DIPs umbilicais leves, quando comparados ao grupo com DIPs umbilicais ausentes. O mecanismo fisiológico que desencadeia a desaceleração variável não envolve a hipoxia fetal, mas simfenômenos de compressão do cordão que promovem uma resposta reflexa do sistema nervoso autônomo normal, podendo ou não estar associado à oligohidramnia ${ }^{21}$, esta sim, relacionada à centralização hipoxêmica da circulação fetal.

$\mathrm{Na}$ avaliação do índice de Apgar de $1^{\circ}$ minuto, constatou-se que a ocorrência de alterações foi significativamente maior nos casos com cardiotocografia de padrão inativo, na ausência de acelerações transitórias e na presença de desacelerações. No presente trabalho, o índice de Apgar no $5^{\circ}$ minuto, apresentou-se alterado em $16 \%$ da população, porém, não se observou associação com os parâmetros cardiotocográficos. Montenegro et al. ${ }^{22}$ avaliam 88 gestantes com DZ ou DR, e encontram Apgar de $5^{\circ}$ minuto inferior a $7 \mathrm{em}$ $17 \%$ dos casos, porém não comparam com os dados cardiotocográficos.

A necessidade de intubação do recémnascido correlacionou-se positivamente com os parâmetros cardiotocográficos. Nem sempre a indicação de intubação é decorrente de intercorrências no período anteparto, mas sim da prematuridade destes fetos, 0 que prejudica a utilização deste parâmetro como fator prognóstico para o desenvolvimento de lesões neurológicas futuras de origem hipoxêmica do período periparto. Tal assertiva tem respaldo nas observações de Weiss et al..$^{23}$ ao compararem $5 \mathrm{I}$ casos de DZ ou DR com o mesmo número de casos com dopplervelocimetria normal, pareados por idade gestacional. Não constatam diferenças significativas na proporção de recém-nascidos que necessitaram de intubação.

Em relação à mortalidade, observou-se correlação dos parâmetros cardiotocográficos com os óbitos neonatais precoces, que se relacionam freqüentemente à asfixia perinatal. Nesta análise surge um fato interessante, queé a correlação positiva entre o padrão denominado pseudo sinusoidal com o óbito. Esta alteração poderia, por conjectura, representar a falência na regulação da FCF por lesões no sistema nervoso central. Neste sentido, Okamura et al. ${ }^{24}$ descrevem padrão cardiotocográfico semelhante ao sinusoidal, denominado por estes autores de padrão "flip flap", e a sua presença correlacionou-se significativamente com a leucomalácia periventricular, considerada uma das lesões neurológicas da hipoxia tecidual fetal ${ }^{25}$.

Amortalidade no berçário apresentou associação com a ausência de acelerações transitórias e com a redução na variabilidade da $\mathrm{FCF}$, fatores estes que podem ser decorrentes não só do sofrimento fetal propriamente dito, como também estar relacionado à prematuridade. Evidentemente, alterações nos parâmetros cardiotocográficos evoluem com morbidade e mortalidade perinatais elevadas ${ }^{26}$, principalmente em casos de DZ ou DR nas artérias umbilicais.

Agravidade da insuficiência placentária em gestações longe do termo preocupa sobremaneira o obstetra, a paciente e seus familiares, pois resultados desfavoráveis passam a constituir real possibilidade na gestação em curso. A associação entre as anormalidades da FCF ea acidemia fetal, demonstradas no presente estudo, respaldam a conduta intervencionista mesmo em situações de extrema prematuridade. Em contrapartida, a manutenção de padrões normais da cardiotocografia permitem o acompanhamento da gravidez, sob vigilância rigorosa da vitalidade fetal por meio deste método propedêutico, que irá auxiliar na condução dos casos de DZ ou DR nas artérias umbilicais.

\section{Conclusão}

Constata-se correlação positiva entre as alterações dos parâmetros da freqüência cardíaca fetal, avaliados pela cardiotocografia anteparto, com os resultados perinatais adversos. A avaliação da vitalidade fetal pela cardiotocografia, em gestações com DZ ou DR nas artérias umbilicais, proporcionará embasamento para as condutas a serem adotadas frente à gravidade da insuficiência placentária.

\section{SUMMARY}

Cardiotocography in pregnancies WITH ABSENT OR REVERSED END-DIASTOLIC VELOCITY IN THE UMBILICAL ARTERIES: ANALISYS OF PERINATAL OUTCOME

OBEECTINES. To study the correlation between cardiotocography parameters and perinatal outcome in pregnancies with absent or reversed enddiastolic velocity (AERDV) in the umbilical arteries.

METHODS. One hundred and twenty-seven cases presenting with AREDV followed between 1993 and 2000 were selected for analysis. The last cardiotocographic tracing performed on the day of delivery was reviewed and the following parameters were considered: magnitude of longterm variability, presence or absence of acceleration, late deceleration, variable deceleration, prolonged deceleration, pseudo sinusoidal pattern and the classification in normal, suspected and abnormal tracing.

RESULTS. A significant $(p<0.05)$ association was found between abnormal tracing and acidemia at birth (pH inferior to 7.20) with $71.9 \%$, first minute Apgar score inferior to seven (73.4\%), newborn intubation at delivery (64. $1 \%)$, and early neonatal death (20.3\%). The absence of acceleration was associated $(p<0.05)$ to acidemia at birth $(58.8 \%)$, first minute Apgar score inferior to seven (67.7\%), newborn intubation at delivery $(5 / \%)$ and neonatal death $(29.4 \%)$. Low FHR variability $(<5 \mathrm{bpm})$ was associated to $(p<0.05)$ : acidemia at birth (88.5\%), newborn intubation at delivery (69.2\%), early neonatal death (34.6\%) and neonatal death $(42.3 \%)$. Late decelerations were significantly $(p<0,05)$ related to acidemia at birth.(78.2\%). The severe variable deceleration was associated to $(p<0.05)$ : acidemia at birth (79.3\%), newborn intubation at delivery (69\%) and early neonatal death (I7.2\%). Prolonged deceleration was associated to $(p<0.05)$ newborn intubation at delivery (70.6\%). Pseudo sinusoidal pattern was associated to $(p<0.05)$ early neonatal death (60\%).

CONCLUSION. The AREDV represents a severe fetal compromise with high risk to neonatal morbidity and mortality, and correlation between cardiotocography abnormalities and adverse perinatal outcome was demonstrated. [Rev Assoc Med Bras 2003; 49(I): 79-85]

KEYWORDS: Absent or reversed end-diastolic velocity. Cardiotocography. Perinatal outcome. 


\section{RefERÊNCIAS}

I. American College of Obstetricians \& Gynecologists (ACOG) practice bulletin. Antepartum fetal surveillance. Practice bulletin number 9, October 1999. Clinical management guidelines for obstetrician-gynecologists. Int J Obstet Gynecol 2000; 68: I 75-86.

2. Trudinger BJ, Giles WB, Cook CM, BombardieriJ, Collins L. Fetal umbilical artery flow velocity waveforms and placental resistance: clinical significance. $\mathrm{Br} J$ Obstet Gynaecol 1985; 92:23-30.

3. Salafia CM, PezzuloJC, Minior VK, Divon MY. Placental pathology of absent and reversed end-diastolic flow in growth-restricted fetuses. Obstet Gynecol I 997; 90:830-6.

4. Brar HS, Platt LD. Reverse end diastolic flow velocity on umbilical artery velocimetry in high risk pregnancies: an ominous finding with adverse pregnancy outcome. Am J Obstet Gynecol 1988; I59:559-6I.

5. Battaglia C, Artini PG, Galli PA, D’Ambrogio G, Droghini F, Genazzani AR. Absent or reversed end-diastolic flow in umbilical artery and severe intrauterine growth retardation. An ominous association. Acta Obstet Gynecol Scand 1993; 72: 167-7I.

6. Karsdorp VHM, Van Vugt JMG, Van Geijn HP, Kostense PJ, Arduini D, Montenegro N, et al. Clinical significance of absent or reversed end diastolic velocity waveforms in umbilical artery. Lancet 1994; 344: 1664-8.

7. Kurkinen-Räty $M$, Kivelä $A$, Jouppila $P$. The clinical significance of an absent end-diastolic velocity in the umbilical artery detected before the $34^{\text {th }}$ week of pregnancy. Acta Obstet Gynecol Scand 1997; 76:398-404.

8. Yamamoto RM, Francisco RPV, Miyadahira S, Chuba CC, Zugaib M. Fatores prognósticos para o óbito perinatal em gestações com diástole zero ou reversa na doppler velocimetria das artérias umbilicais. Rev Bras Ginecol Obstet 2000; 22:353-63.

9. Black RS, Campbell S. Cardiotocography versus Doppler. Ultrasound Obstet Gynecol 1997; 9:| 48-5I.

10. Baschat AA, Weiner CP. Umbilical artery Doppler screening for detection of the small fetus in need of antepartum surveillance. Am J Obstet Gynecol 2000; 182: I54-8.

II. Haley J, Tuffnell DJ, Johnson N. Randomized controlled trial of cardiotocography versus umbilical artery Doppler in the management of small for gestational age fetuses. Br J Obstet Gynaecol I 997; 104:43 I-5.

12. Zugaib M, Forsythe AB, Nuwayhid B, LiebSM, Tabsh K, Erkolla R, et al. Mechanisms of beatto-beat variability in the heart rate of the neonate lamb. II. Effects of hypoxia. Am J Obstet Gynecol 1980; 138:453-8.

13. Zugaib M. Contribuição ao estudo do controle autonômico das funções cardiovasculares fetais e neonatais (dissertação). São Paulo: Faculdade de Medicina, Universidade de São Paulo; 1980.

14. Flynn AM, KellyJ. Evaluation of fetal well-being by antepartum fetal heart rate monitoring. $\mathrm{Br}$ Med J 1977; I:936-9.

15. National Institute of Child Health and Human Development Research Planning Workshop. Electronic fetal heart rate monitoring: research guidelines for interpretation. Am J Obstet Gynecol 1997; 177: 1385-90.

16. Zugaib M, Behlel. Cardiotocografia anteparto de repouso. I-Considerações sobre conceito, metodologia e interpretação. Proposição de índice cardiotocométrico. Rev Bras Ginecol Obstet 1981; :72-85.

17. Zelop CM, Richardson DK, Heffner LJ. Outcomes of severely abnormal umbilical artery Doppler velocimetry in structurally normal singleton fetuses. Obstet Gynecol 1996; 87:434-8.
18. Hanson MA. Do we now understand the control of the fetal circulation? Eur J Obstet Gynecol Reprod Biol 1997; 75:55-6I.

19.Ikeda T, Murata Y, Quilligan EJ, Parer JT, Theunissen IM, Cifuentes $P$, et al. Fetal heart rate patterns in postaphyxiated fetal lambs with brain damage. Am J Obstet Gynecol 1998; 179:1329-37.

20. Nomura RMY, Francisco RPV, Miyadahira S, Zugaib M. Modelo matemático para predição da acidose metabólica no nascimento em gestações com diástole zero ou reversa. Rev Bras Ginecol Obstet 2002; 24:26I -9.

21. Keegan Jr KA. The nonstress test. Clin Obstet Gynecol 1987; 30:921-35.

22. Montenegro N, Santos F, Tavares E, Matias A, Barros H, Leite LP. Outcome of 88 pregnancies with absent or reversed end-diastolic blood flow (ARED flow) in the umbilical arteries. Eur J Obstet Gynecol Reprod Biol 1998; 79:43-6.

23. Weiss E, Ulrich S, Berle P. Condition at birth of infants with previously absent or reverse umbilical artery end-diastolic flow velocities. Arch Gynecol Obstet 1992; 252:37-43.

24. Okamura M, ItakuraA, Kurauchi O, HayakawaF, MizutaniS, Tomoda Y. Fetal heart rate patterns associated with periventricular leukomalacia. Int] Gynecol Obstet 1997; 56: 13-8.

25. MurotsukiJ, Bocking AD, Gagnon R. Fetal heart rate patterns in growth-restricted fetal sheep induced by chronic fetal placental embolization. Am J Obstet Gynecol 1997; 176:282-90.

26. WestgateJA, Gunn AJ, Gunn TR. Antecedents of neonatal encephalopathy with fetal acidaemia at term. BrJ Obstet Gynaecol I999; 106:774-82.

Artigo recebido: 05/04/2002 Aceito para publicação: 09/08/2002 